\title{
Strain-induced optical anisotropy in self-organized quantum structures at the $E_{1}$ transition
}

\author{
J. A. Prieto, ${ }^{\text {a) }}$ G. Armelles, C. Priester, ${ }^{\text {b) }}$ J. M. García, ${ }^{c}$ L. González, and R. García \\ Instituto de Microelectrónica de Madrid (CNM, CSIC), Isaac Newton 8, 28760, \\ Tres Cantos (Madrid), Spain
}

(Received 7 December 1999; accepted for publication 17 February 2000)

\begin{abstract}
In-plane optical anisotropies of (001)-oriented InAs/InP self-assembled quantum wires and dots structures are studied by means of photoreflectance in the spectral region of the $E_{1}$ transition of bulk InAs. The energy position of the transition observed in the quantum wires depends on the light polarization; quantum dots do not exhibit, in contrast, such an optical anisotropy. This anisotropy is attributed to the splitting of the four-fold degenerate $E_{1}$ transition produced by the strong triaxial behavior of the strain that appears in wires and not in dots. (C) 2000 American Institute of Physics. [S0003-6951(00)00616-1]
\end{abstract}

After the development of the lattice-matched quantum well $(\mathrm{QW})$ structure and the introduction of strained well layers by means of the heteroepitaxial growth, additional advance in semiconductor science has been achieved via further reduction of dimensionality, i.e., obtaining quantum wire (QWR) and quantum dot (QD) structures. The most suitable approach to these structures from the technological point of view, due to its feasibility and damage-free nature, could be the one based on the selforganization process. This process takes place during the heteroepitaxial growth and is the consequence of the elastic relaxation of the strain caused by the lattice mismatch between epilayer and substrate. Surprisingly, although self-organized QDs were obtained for the first time more than one decade ago and their basics are well understood, ${ }^{1}$ work on self-organized QWRs is very recent and scarce, ${ }^{2-5}$ their formation being object of controversy and their physics remaining unexplored.

Magnetophotoluminescence investigations in strained QWRs fabricated through electron-beam lithography and wet etching on (001) substrates reveal that such structures exhibit a strongly anisotropic strain. ${ }^{6,7}$ This anisotropic strain causes large behavior deviations with respect to biaxially strained QWs. For instance, due to its symmetry-breaking effect on the hole wave functions, ${ }^{7}$ photoluminescence signal shows an important strain-induced in-plane optical anisotropy. However, quantum confinement and strain effects are often mixed so that they are difficult to distinguish.

The strain effect is expected to be more easily observable in the $E_{1}$ transition than in the $E_{0}$ one. As a matter of fact, the large effective masses at the $L$ point of the Brillouin zone make the quantum confinement effect small ${ }^{8}$ and sometimes negligible ${ }^{9}$ for typical nanostructure dimensions. On the other hand, anisotropic strain (contrary to the biaxial strain of strained QWs) may break the four-fold degeneracy of the $E_{1}$ transition. This could result in a large in-plane optical anisotropy similar to that found in lattice-matched

\footnotetext{
${ }^{\text {a) }}$ Electronic address: josea@imm.cnm.csic.es

${ }^{b)}$ Institut d'Electronique et de Microélectronique du Nord, Département ISEN, BP 69, 59652 Villeneuve d'Ascq cedex, France. UMR CNRS8520.

${ }^{c)}$ Electronic mail: jorgem@imm.cnm.csic.es
}

QWs and superlattices grown on lowsymmetry oriented substrates. ${ }^{10,11}$ In this letter we present a polarization study of this transition both in InAs self-organized QWRs and QDs grown on (001) InP substrates. Large in-plane optical anisotropy is found in QWRs, which appears as an energy difference or splitting of the QWR-related $E_{1}$-like transition when measured using light polarized parallel or perpendicular to the QWR axis. On the contrary, no significant in-plane optical anisotropy is observable in QDs. The splitting is caused by the strong shear component of the strain that appears in our wires. Calculations within the finite difference method and deformation potential theory together with the experimental results allow us to estimate the intervalley deformation potential of bulk InAs at $L\left(D_{1}^{5}\right)$.

InAs self-organized QDs and QWRs were obtained on nominally flat (001) InP substrates by means of molecular beam epitaxy (MBE). InAs is deposited under the same conditions in both cases, being the substrate temperature, arsenic pressure and growth rate respectively $400{ }^{\circ} \mathrm{C}, 2 \times 10^{-6}$ mbar and 0.5 ML/s. Once the InAs deposition is finished, an annealing under arsenic flux at a substrate temperature of $480^{\circ} \mathrm{C}$ takes place for $10-20 \mathrm{~s}$. As the reflection highenergy electron diffraction pattern indicates, the twodimensional to three-dimensional transition occurs during the annealing. The appearance of QDs or QWRs crucially depends on the manner in which the buffer layer is grown. ${ }^{5}$ Thus, when atomic-layer MBE (ALMBE) is used, isolated QDs form. On the contrary, MBE gives rise to the systematic formation of surface roughness with features preferentially aligned along the [110] direction, which establishes the longitudinal axis of the QWRs that form afterwards.

Figure 1 shows representative atomic force microscopy (AFM) images of samples containing QDs (a) and QWRs (b) structures. QDs show a slight elongation along the [110] direction. The wires observed in the QWR sample have a quite regular size, being the length of the wires usually larger than $1 \mu \mathrm{m}$.

The polarization study of the $E_{1}$ transition of QDs and QWRs was carried out at $80 \mathrm{~K}$ via photoreflectance (PR). Incident light was polarized along the directions parallel and perpendicular ([110] and [110], respectively) to the QWR 


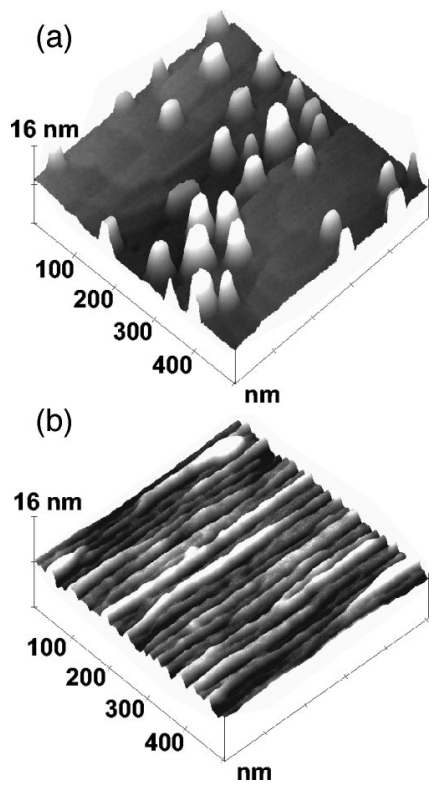

FIG. 1. Representative AFM images of InAs QD (a) and QWR (b) structures grown under similar conditions on nominally flat (001) InP substrates. The InAs deposition amounts to 3.5 ML in the former and 4.5 ML in the latter, whereas the buffer layer was deposited using, respectively, ALMBE and MBE.

axis. Figure 2 displays PR spectra from the samples of Fig. 1. QDs [Fig. 2(a)] exhibit a feature, close to $2.65 \mathrm{eV}$, associated with the $E_{1}$ critical point of bulk InAs. ${ }^{8}$ QWRs also exhibit this feature [Fig. 2(b)], but the energy position of this feature depends on the light polarization. The intensity of the feature observed does not depend on light polarization neither in QDs nor in QWRs.

A simple Krönig-Penney model, applied to the $L$ point of the Brillouin zone, has been used to explain the behavior of the $E_{1}$ transition in lattice-matched QWs and superlattices. ${ }^{12,13}$ However, the effect of strain on the band structure has to be taken into account previously when strained nanostructures are considered. The four $L$ interband valleys, corresponding to the [111], [ $\overline{1} \overline{1} 1],[1 \overline{1} \overline{1}]$, and [111] directions, give rise to the four-fold degenerate $E_{1}$ transition. (001) biaxial strain $\left[\varepsilon_{x x}=\varepsilon_{y y}=\varepsilon_{\|} ; \quad \varepsilon_{z z}=\varepsilon_{\perp}\right.$

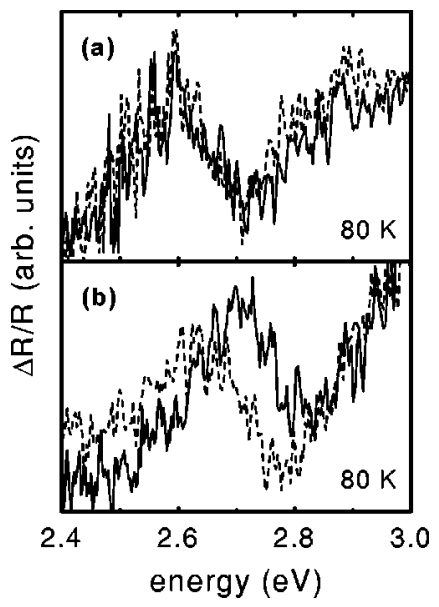

FIG. 2. $80 \mathrm{~K}$ PR spectra of the samples of Fig. 1 showing the QD (a) and QWR (b) related $E_{1}$-like transition of the QDs (a) and the QWRs (b). The incident light beam was polarized along the [1110] (solid line) and [110]

(dashed line) in-plane perpendicular directions. and the other purely shear-like (nondiagonal ones). The
Downloaded 26 Feb 2010 to 161.111 .180 .191 . Redistribution subject to AIP license or copyright; see http://apl.aip.org/apl/copyright.jsp
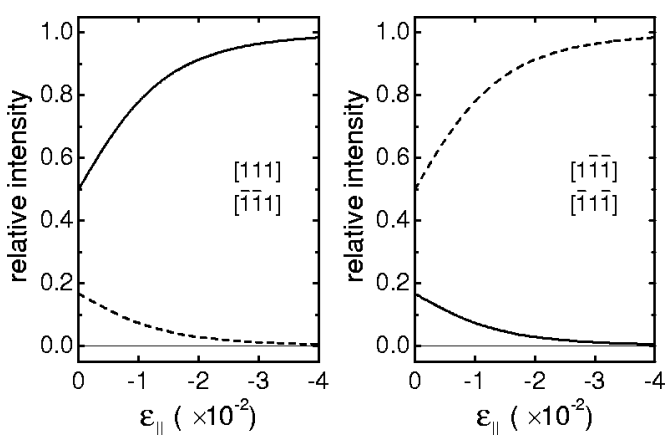

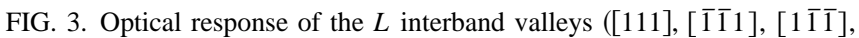
and $[\overline{1} 1 \overline{1}]$ ) in the $[\overline{1} 10]$ (solid line) and [110] (dashed line) directions calculated for a (001) biaxially strained zinc-blende semiconductor as a function of the in-plane strain component $\left(\varepsilon_{\|}\right)$.

$\left.=-2\left(C_{12} / C_{11}\right) \varepsilon_{\|} ; \varepsilon_{x y}=\varepsilon_{x z}=\varepsilon_{y z}=0\right]$, due to its high symmetry, modifies the energy, but does not remove the degeneracy of this transition. Nevertheless, its shear part induces intravalley interactions between the valence band states ${ }^{14,15}$ and, in consequence, modifications in the oscillator strength. Figure 3 shows the relative intensities of the four energetically equivalent $E_{1}$ transitions versus the in-plane strain component $\left(\varepsilon_{\|}\right)$calculated for the [110] and [110] polarization. The intraband deformation potentials was taken to be $D_{3}^{3}=-4.3 \mathrm{eV}$, and the spin-orbit splitting $\Delta_{1}=0.22 \mathrm{eV}$. As can be observed, when $\varepsilon_{\|}$is similar to the lattice mismatch between InAs and InP (3.1\%), the optical response of the $L$ interband valleys is completely polarized along either [ $\overline{1} 10]$

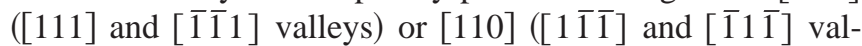
leys). Since the degeneracy is not removed and the relative intensities of the different contributions to the signal are the same, (001) biaxial strain should not cause any in-plane optical anisotropy in the $E_{1}$ transition.

QWRs are strained to the substrate along their axis, but they may be elastic relaxed in the directions perpendicular to the wire axis. Figure 4 presents cross-section strain mappings corresponding to an InAs/InP QWR like those of the sample in Fig. 1. These strain mappings has been obtained within the finite difference method assuming infinite length and display the values of the strain components, referred to the basis characteristic of the QWR $\left(\left\{x^{\prime}, y^{\prime}, z^{\prime}\right\}=\{[110]\right.$, $[\overline{1} 10],[001]\})$, in the $\{\overline{1} 10\}$ plane, perpendicular to the QWR axis. The $\varepsilon_{x^{\prime} y^{\prime}}$ and $\varepsilon_{y^{\prime} z^{\prime}}$ components have negligible values and for simplicity only $\varepsilon_{x^{\prime} x^{\prime}}$ and $\varepsilon_{x^{\prime} z^{\prime}}$ mappings are shown. In general, elastic relaxation becomes important only at the QWR edges, i.e., strain varies little in its central region. Notice that the $\{110\}$ plane is an inversion plane for $\varepsilon_{x^{\prime} z^{\prime}}$. In the $\{x, y, z\}=\{[100],[010],[001]\}$ basis the strain components satisfy the relationships

$$
\begin{aligned}
& \varepsilon_{x x}=\varepsilon_{y y}=\left(\varepsilon_{x^{\prime} x^{\prime}}+\varepsilon_{y^{\prime} y^{\prime}}\right) / 2, \\
& \varepsilon_{z z}=\varepsilon_{z^{\prime} z^{\prime}}, \\
& \varepsilon_{x y}=\left(\varepsilon_{x^{\prime} x^{\prime}}-\varepsilon_{y^{\prime} y^{\prime}}\right) / 2, \\
& \varepsilon_{x z}=\varepsilon_{y z}=\varepsilon_{x^{\prime} z^{\prime}} / \sqrt{ } 2 .
\end{aligned}
$$

Therefore, the strain state of a self-organized QWR consists of two parts, one (001) biaxial-like (diagonal components) and the other purely shear-like (nondiagonal ones). The 

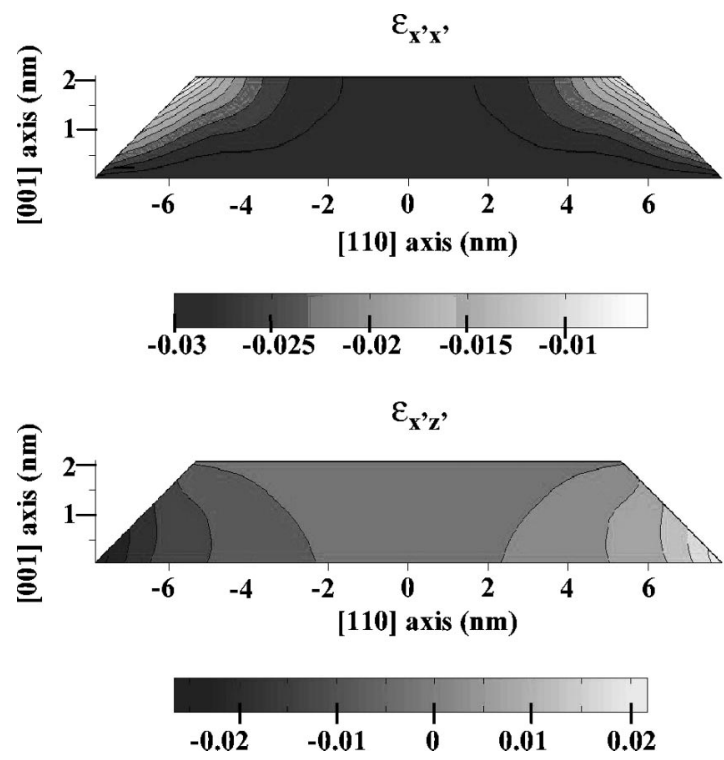

FIG. 4. Cross-section strain mappings showing different strain components, referred to the basis defined by the QWR axis $\left(\left\{x^{\prime}, y^{\prime}, z^{\prime}\right\}\right.$ $=\{[110],[\overline{1} 10],[001]\})$, in an InAs QWR $15 \mathrm{~nm}$ wide (at base), $2 \mathrm{~nm}$ high, and faceted by [001] and [113] planes. The dimensions of the InAs wire were taken from transmission electron microscopy pictures (Ref. 5).

(001) biaxial-like part, as commented before, maintains the degeneracy of the $E_{1}$ transition, but modifies the optical response of the $L$ interband valleys in the manner indicated in Fig. 3. The purely shear-like part also produces intravalley interactions. ${ }^{14,15}$ However, these interactions are negligible compared with those caused by the biaxial-like part as the nondiagonal components are much smaller than the diagonal ones. In addition, the purely shear-like part leads to a breaking of degeneracy and, as a result, to the splitting of the $E_{1}$ transition. This effect is described in terms of the intervalley deformation potential $D_{1}^{5}$ as indicated in Table I. In principle, not one but three $E_{1}$-like features may be observable in self-organized QWRs because of anisotropic strain. Two of them ([111] and [ $\overline{1} \overline{1} 1])$ are polarized along the QWR axis, whereas the others $([1 \overline{1} \overline{1}]$ and $[\overline{1} \overline{1}])$ along the perpendicu-

TABLE I. Splitting terms of the $L$ interband valleys due to the purely shear-like part of the anisotropic strain characteristic of QWRs. $D_{1}^{5}$ is the intervalley deformation potential, whereas $\varepsilon_{x y}$ and $\varepsilon_{x z}$ are strain components expressed in the $\{x, y, z\}=\{[100],[010],[001]\}$ basis.

\begin{tabular}{cc}
\hline \hline Interband valley & $\Delta E_{1}$ \\
\hline$[111]$ & $\left(D_{1}^{5} / \sqrt{ } 3\right)\left(\varepsilon_{x y}+2 \varepsilon_{x z}\right)$ \\
{$[\overline{1} \overline{1} 1]$} & $\left(D_{1}^{5} / \sqrt{ } 3\right)\left(\varepsilon_{x y}-2 \varepsilon_{x z}\right)$ \\
{$[1 \overline{1} \overline{1}]$} & $-\left(D_{1}^{5} / \sqrt{3}\right) \varepsilon_{x y}$ \\
{$[\overline{1} 1 \overline{1}]$} & $-\left(D_{1}^{5} / \sqrt{ } 3\right) \varepsilon_{x y}$ \\
\hline \hline
\end{tabular}

lar direction, all of them showing the same relative intensity. Nevertheless, according to the picture given by the KrönigPenney model, wave functions are expected to be localized mainly in the QWR central region. Here, the mean value of $\varepsilon_{x z}$ is zero as the $\{110\}$ plane is an inversion plane for this strain component. Therefore, instead of three features only two should be observable in self-organized QWRs. The strain-induced energy difference between these features or splitting is given by

$$
\Delta E_{1}\left(\varepsilon_{x y}\right)=2\left(D_{1}^{5}\right) \varepsilon_{x y} / \sqrt{ } 3 .
$$

Due to the large QWR length and width compared to its height the confining direction should be mainly the [001] one, and therefore, anisotropic strain must be the principal cause of splitting so that the observed optical anisotropy is a measure of the mean value of $\varepsilon_{x y}$, i.e., the anisotropic elastic relaxation in the growth plane. Moreover, in view of Fig. 3 both transitions should exhibit the same oscillator strength, as is experimentally observed. From the experimental splitting of the $E_{1}$ and the theoretical calculation of $\varepsilon_{x y}, D_{1}^{5}$ for InAs can be estimated to be $30 \mathrm{eV}$.

In conclusion, the anisotropic strain of self-assembled QWRs has been found to cause large in-plane optical anisotropy by means of the splitting of the $E_{1}$ transition into two new features. Contrary to the QWRs, self-assembled QDs do not exhibit any splitting.

This work has received financial support by the Spanish CICYT under Grant Nos. MAT95-0966-C02-01 and TIC1020-C02-01.

${ }^{1}$ For an overview, see D. Bimberg, M. Grundmann, and N. N. Ledentsov, Quantum Dot Heterostructures (Wiley, New York, 1999).

${ }^{2}$ B. J. Ohlsson and M. S. Miller, J. Cryst. Growth 188, 387 (1998).

${ }^{3}$ F.-Q. Liu, Z.-G. Wang, B. Xu, and J.-J. Qian, Phys. Lett. A 249, 555 (1998).

${ }^{4}$ A. Subekti, M. J. Paterson, E. M. Goldys, and T. L. Tansley, Appl. Surf. Sci. 140, 190 (1999).

${ }^{5}$ L. González, J. M. García, R. García, C. Ballesteros, J. Martínez-Pastor, and F. Briones, Appl. Phys. Lett. 76, 1104 (2000).

${ }^{6}$ M. Notomi, J. Hammersberg, H. Weman, S. Nojima, H. Sugiura, M. Okamoto, T. Tamamura, and M. Potemski, Phys. Rev. B 52, 11147 (1995).

${ }^{7}$ N. Notomi, J. Hammersberg, J. Zeman, H. Weman, M. Potemski, H. Sugiura, and T. Tamamura, Phys. Rev. Lett. 80, 3125 (1998).

${ }^{8}$ J. A. Prieto, G. Armelles, J. Groenen, and R. Carles, Appl. Phys. Lett. 74, 99 (1999).

${ }^{9}$ J. A. Prieto, G. Armelles, T. Utzmeier, F. Briones, J. C. Ferrer, F. Peiró, A. Cornet, and J. R. Morante, Phys. Rev. Lett. 80, 1094 (1998).

${ }^{10}$ J. A. Prieto and G. Armelles, Phys. Rev. B 53, 6912 (1996).

${ }^{11}$ U. Schmid, N. E. Christensen, M. Cardona, F. Lukes, and K. Ploog, Phys. Rev. B 45, 3546 (1992).

${ }^{12}$ E. E. Mendez, L. L. Chang, G. Landgren, R. Ludeke, L. Esaki, and F. H. Pollak, Phys. Rev. Lett. 46, 1230 (1981).

${ }^{13}$ J. M. Rodríguez, G. Armelles, and F. Briones, Solid State Commun. 67, 859 (1988).

${ }^{14}$ M. Chandrasekhar and F. H. Pollak, Phys. Rev. B 15, 2127 (1977).

${ }^{15}$ F. H. Pollak and M. Cardona, Phys. Rev. 172, 816 (1968). 